\title{
The Pulmonary Metastasectomy in Colorectal Cancer (PulMiCC) trial results cast doubt upon benefit of lung and liver metastasectomy at the time colorectal resection
}

\author{
Norman R. Williams ${ }^{1}$ (D) $\cdot$ Tom Treasure ${ }^{2}$ (D) Fergus Macbeth $^{3}$ (]) \\ Received: 7 December 2021 / Accepted: 17 December 2021 / Published online: 3 January 2022 \\ (c) The Author(s), under exclusive licence to Springer-Verlag GmbH Germany, part of Springer Nature 2022
}

The report by Dr. Tanaka and colleagues of 3-year overall and relapse-free survival following resection of colorectal cancer (CRC) in patients who had both hepatic and pulmonary metastases at the time of diagnosis [1] deserves careful reading and thoughtful analysis. We appreciated the courteous reference to preliminary results of PulMiCC (Pulmonary Metastasectomy in Colorectal Cancer). They correctly noted that this randomised controlled trial (RCT) "indicated that survival benefit of pneumonectomy for patients with pulmonary metastases is likely to be less than is currently assumed".

The operations on their 26 patients up December 2018 relied on the widely held belief that lung metastasectomy improved survival. The prospective PulMiCC cohort study, with data from 512 patients collected to trials standards, has now been published [2] along with a nested RCT in 93 patients [3]. The patients had a previous resection of a primary CRC and were found to have one or more asymptomatic lung nodules and were potential candidates for metastasectomy. After fully informed written consent under clinical trial conditions, 28 were excluded because the lung nodules were either benign or non-CRC neoplasms. The clinical teams selected 263 patients to have metastasectomy, 128 had an elective decision to not have metastasectomy and 93 were randomised.

Tom Treasure

tom.treasure@gmail.com

1 Surgical and Interventional Trials Unit (SITU), University College London, London, UK

2 Clinical Operational Research Unit (CORU), University College London, London, UK

3 Centre for Trials Research, Cardiff University, Cardiff, UK
The survival of the elective patients is shown in the upper panel of Fig. 1. For elective metastasectomy patients, 5 -year survival was $58.5 \%$ (95\% CI: 52.0-64.8) in line with the best results in follow-up studies. This confirms that the PulMiCC cohort replicated "real-world" practice. For those selected to not have metastasectomy, survival was $24.0 \%$ (95\% CI: $16.9-31.9)$ which is very significantly better $(P<0.001)$ than the widely assumed zero. All baseline factors collected solitary versus multiple metastases, non-elevated carcinoembryonic antigen (CEA), absence of liver involvement, age, performance status and lung function - favoured better survival in the metastasectomy patients [2].When all factors were balanced in the RCT (lower graph in Fig. 1), there was no difference at any time point [3]. A small long-term difference due to an occasional true "cure" cannot be excluded, but it is far smaller than believed.

Metastasectomy had a detrimental effect on quality of life [4]. Scores in five dimensions of health utility - mobility, self-care, usual activity, pain and discomfort, anxiety and depression - in EQ-5D-3L fell similarly in the two arms of the RCT [5].

In the report by Tanaka et al. [1], the differences in CEA and the number and size of metastases in their Table 2 would largely explain the difference in survival and the use of the word "curative" is misleading. On a point of terminology, with rare exceptions, lung metastasectomy involves wedge resection, segmentectomy or, at most, lobectomy. The word pneumonectomy is reserved in the thoracic surgery for removal of a whole lung, rarely done for metastases, even by true believers.

It is important to state that not everything in surgery requires an RCT which is a learning point from this paper. Eradication and/or appropriate local control of primary CRC spares suffering and saves lives in a way that is mechanistically obvious and meets the criteria set out by Glasziou and colleagues for interventions that do not require an RCT [6]. But metastatic cancer includes disease below the resolution 
Fig. 1 Product-Limit survival estimates with number at risk and $95 \%$ confidence limits for the prospective cohort of 391 patients (above) and the RCT of 93 patients (below). In the prospective cohort, 263 patients had an elective metastasectomy (red) and 128 did not (blue). All baseline oncological and performance factors favoured the metastasectomy group (see text) which more than halved their risk of death. In the RCT, all these factors were balanced and there was no difference in survival

\section{Survival probability}

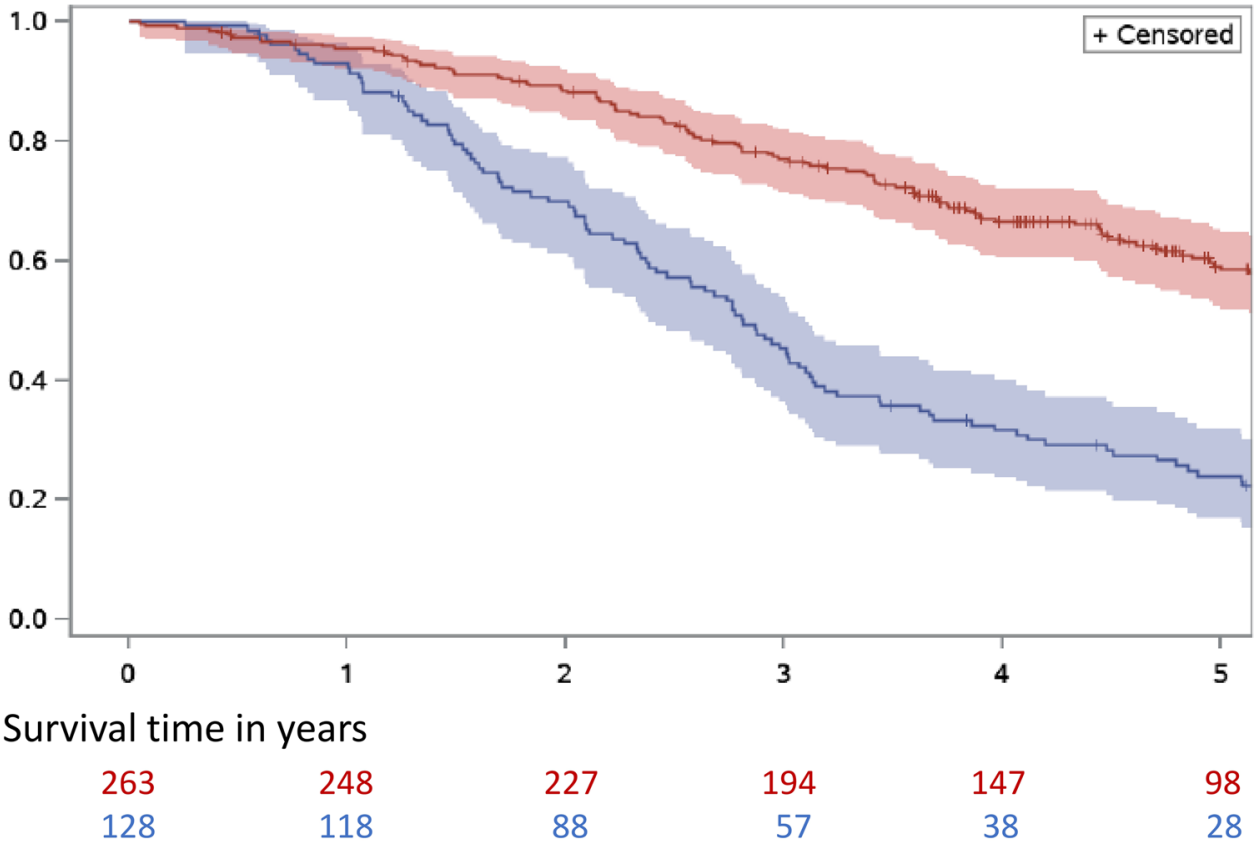

\section{Survival probability}

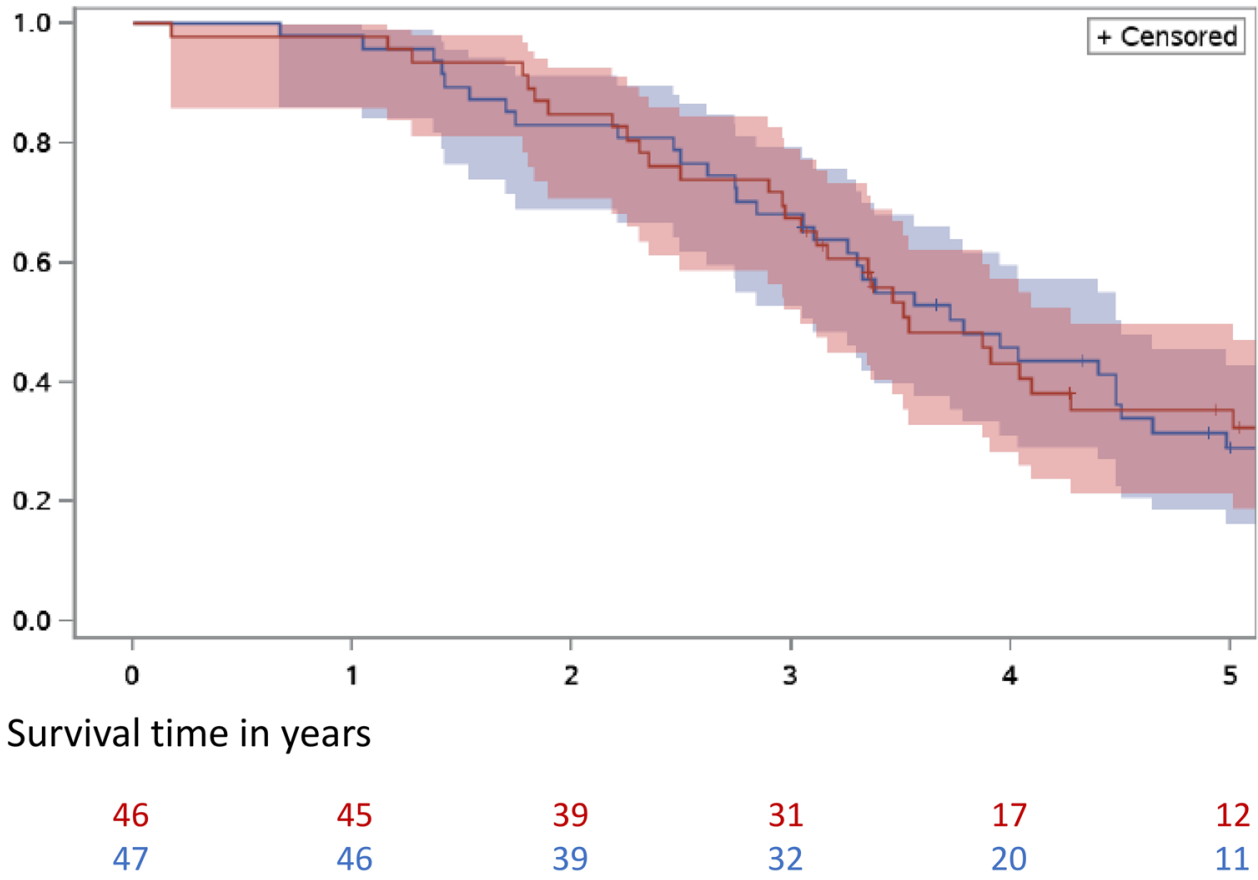

of the best modern imaging, and survival is determined by multiple factors including the inherent biology of the cancer. Operating on those with a panoply of favourable factors gives the impression of benefit from the intervention, but this benefit is largely due to well-informed selection of naturally longer survivors.
Funding This work was supported by Cancer Research UK Grant No. C7678/A11393.

\section{Declarations}

Conflict of interest The authors declare no competing interests. 


\section{References}

1. Tanaka Y, Yamaoka Y, Shiomi A, Kagawa H, Hino H, Manabe $S$ et al (2021) Clinical outcomes following colorectal resection of colorectal cancer with simultaneous hepatic and pulmonary metastases at the time of diagnosis. Langenbecks Arch Surg

2. Treasure T, Farewell V, Macbeth F, Batchelor T, Milosevic M, King J et al (2021) The Pulmonary Metastasectomy in Colorectal Cancer cohort study: analysis of case selection, risk factors and survival in a prospective observational study of 512 patients. Colorectal Dis 23(7):1793-1803

3. Milosevic M, Edwards J, Tsang D, Dunning J, Shackcloth M, Batchelor T et al (2020) Pulmonary Metastasectomy in Colorectal Cancer: updated analysis of 93 randomized patients - control survival is much better than previously assumed. Colorectal Dis 22(10):1314-1324
4. Treasure T, Farewell V, Macbeth F, Monson K, Williams NR, Brew-Graves C et al (2019) Pulmonary Metastasectomy versus Continued Active Monitoring in Colorectal Cancer (PulMiCC): a multicentre randomised clinical trial. Trials 20(1):718

5. Brew-Graves C, Farewell V, Monson K, Milosevic M, Williams NR, Morris E et al (2021) Pulmonary metastasectomy in colorectal cancer: health utility scores by EQ-5D-3L in a randomized controlled trial show no benefit from lung metastasectomy. Colorectal Dis 23(1):200-205

6. Glasziou P, Chalmers I, Rawlins M, McCulloch P (2007) When are randomised trials unnecessary? Picking signal from noise BMJ 334(7589):349-351

Publisher's note Springer Nature remains neutral with regard to jurisdictional claims in published maps and institutional affiliations. 